\title{
Metastatic Follicular Thyroid Carcinoma Presenting as Thoracic Cord Compression
}

\author{
Ryan Petruccia, b, Karrar Bohreh ${ }^{\mathrm{a}}$, Navin Niles ${ }^{\mathrm{a}}$
}

\begin{abstract}
Follicular thyroid carcinoma (FTC) is an uncommon cancer and the incidence of FTC is higher in endemic areas of iodine deficiency or endemic goiter. Up until the 1990s Fiji was listed as an iodine deficient country. We report a rare case of a 53-year-old native Fijian man who presented to our hospital with spinal cord compression due to a metastatic deposit of an undiagnosed FTC. He underwent emergent neurosurgical treatment for his cord compression, with histology of the lesion at the level of T5 identifying metastatic FTC. Despite the emergent surgery, he did not have any neurological recovery. Total thyroidectomy confirmed the presence of a large left-sided FTC and the patient was assessed for radioactive iodine treatment. Nuclear medicine imaging revealed extensive distant bony metastatic disease. Unfortunately due to his significant distant disease burden, he was unable to undergo radioactive iodine ablation therapy. After significant allied health input he was discharged home with community palliative care input.
\end{abstract}

Keywords: Metastatic follicular thyroid cancer; Metastatic follicular thyroid carcinoma; Cord compression

\section{Introduction}

Thyroid carcinoma is an uncommon cancer and comprises of five histological subtypes: papillary, follicular, medullary, poorly differentiated and undifferentiated. The two main differentiated subtypes are papillary and follicular with papillary having favorable long-term survival rates [1]. Follicular thyroid carcinoma (FTC) has a higher propensity to metastasize and thus a worse prognosis [2].

The World Health Organization (WHO) defines FTC as "A malignant epithelial tumour showing follicular differentiation

Manuscript submitted January 24, 2021, accepted February 1, 2021

Published online March 5, 2021

aLiverpool Hospital Department of Endocrine Surgery, Head and Neck Surgery, Elizabeth Street, Liverpool, NSW 2170, Australia

${ }^{b}$ Corresponding Author: Ryan Petrucci, Liverpool Hospital Department of Endocrine Surgery, Head and Neck Surgery, Elizabeth Street, Liverpool, NSW 2170, Australia. Email: r_petrucci@hotmail.com

doi: https://doi.org/10.14740/jmc3658 and lacking the diagnostic nuclear features". FTC accounts for $10-20 \%$ of differentiated thyroid carcinomas. Demographically it affects women 3:1 compared to men and usually presents in fifth to seventh decade [3]. FTC demonstrates vascular invasion and so usually metastasizes to both bone and lung mostly without nodal spread [4].

Although thyroid cancer is well known to metastasize to bone, including vertebrae, cord compression is very rare as an initial presentation for the carcinoma and our literature search could only locate nine documented cases. Treatment of FTC usually involves a multi-disciplinary team (MDT) approach including surgical resection, hormonal therapy and radioactive iodine ablation therapy (RAI). We present the case of a patient who presented to hospital with symptoms of spinal cord compression due to an undiagnosed metastatic FTC.

\section{Case Report}

A 53-year-old man presented to our emergency department with several weeks of progressive leg weakness, constipation and difficulty in voiding urine. On further questioning he admitted 1 year's history of mild mid-back pain and occasional neck pain. He had no significant past medical history. Neurological examination demonstrated he had reduced power to the lower limbs bilaterally and decreased sharp and light touch sensation to the lower limbs with hyperreflexia. Additionally he had decreased sensation bilaterally up to the T7 dermatome. Urgent computed tomography (CT) spine showed T5 cord compression from a lytic T5 lesion. Magnetic resonance imaging (MRI) showed a T5 vertebral body mass (Figs. 1, 2) with multiple other enhancing foci within the spine, a destructive lesion in right iliac crest and a left-sided neck mass (Fig. 3).

The patient underwent urgent T5 laminectomy with T3-7 fixation. Post-operatively the patient underwent targeted radiotherapy to the spinal lesion site as well as the right iliac fossa mass. Despite urgent surgery and radiotherapy, the patient did not show signs of neurological recovery. Post-surgery and post-radiotherapy, he lost power to his lower limbs, became double incontinent and became wheelchair bound.

Histology of the T5 mass was reported as metastatic FTC.

Endocrine surgeons were consulted and a CT of neck was performed. This revealed a large left-sided thyroid mass with deviation of trachea. Further to his CT, the patient underwent a bone and fluorodeoxyglucose-positron emission tomography (FDG-PET) scan. He underwent a total thyroidectomy and left central neck dissection on day 26 of his admission. Pathology 


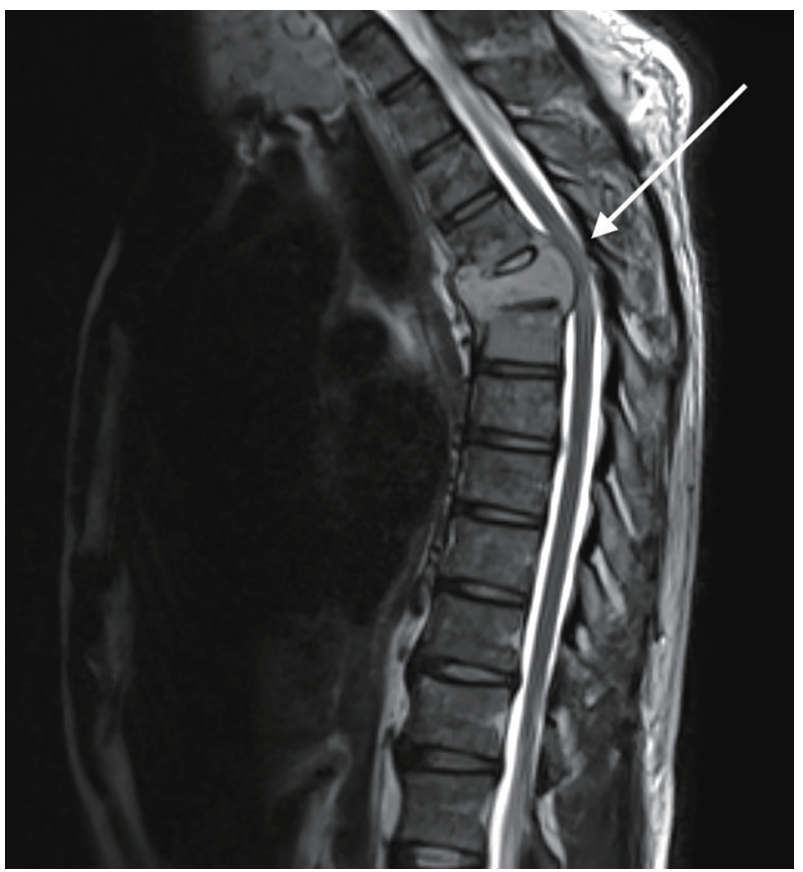

Figure 1. Sagittal magnetic resonance imaging (MRI) of the thoracic spine demonstrating a severe attenuation of the T5 thoracic cord with increased abnormal T2 signal (highlighted by the arrow).

was reported as a left-sided $90 \times 70 \times 70 \mathrm{~mm}$ sized moderately differentiated FTC with vascular invasion and nodal spread.

During his post-operative recovery, he was evaluated for RAI treatment. It was deemed that the large right iliac crest metastasis would greatly reduce the efficacy of RAI for his other metastatic disease, so the patient was referred to a specialist orthopedic MDT meeting for surgical resection assessment. Unfortunately the mass could not be safely operated on due to significant neovascularization and the patient underwent cryotherapy embolization via interventional radiology

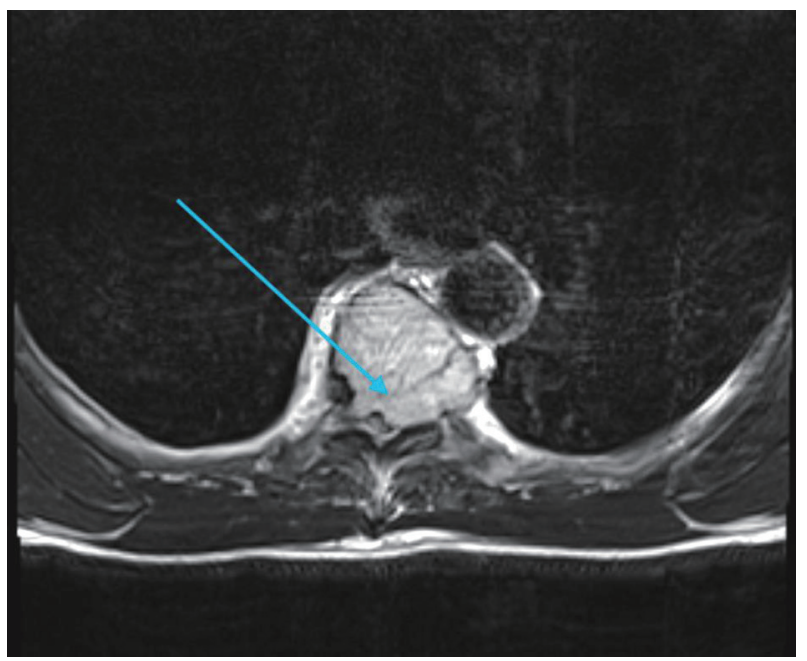

Figure 2. Axial view magnetic resonance imaging (MRI) of T5 vertebrae demonstrating large lytic lesion causing cord compression (highlighted by the arrow).

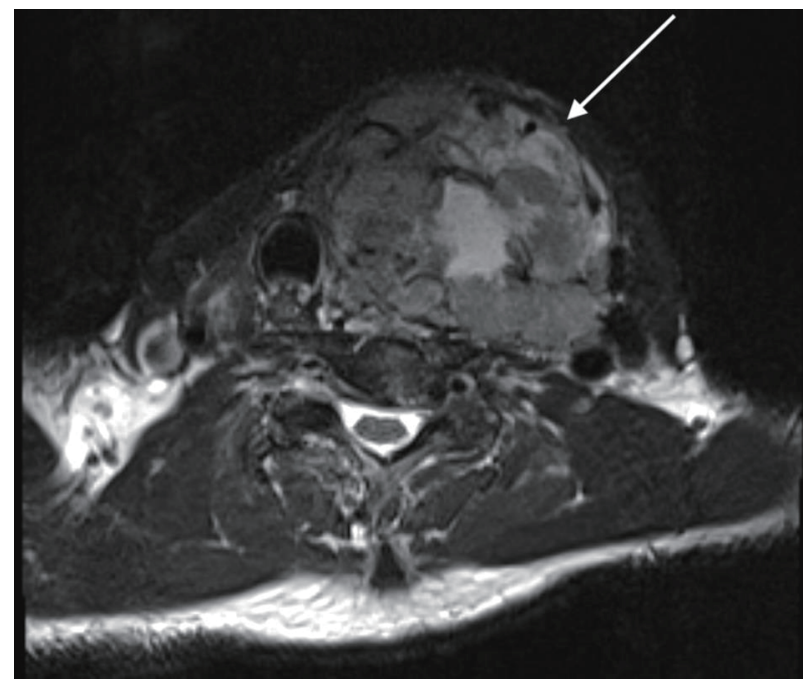

Figure 3. Axial magnetic resonance imaging (MRI) of the neck. There is a large cervical soft tissue mass, with the left thyroid lobe as the focal point measuring $6.3 \times 7.3 \times 10 \mathrm{~cm}$ (highlighted by the arrow) with deviation of the trachea.

in an attempt to reduce the size and metabolic activity of the metastasis.

Subsequent repeat bone scans showed new areas of macroscopic spread including those in the sternum and the base of the lung, as well as an increase in the size of the iliac metastasis despite embolization. Nuclear medicine was consulted throughout his admission, and after review the decision was made that the patient would not benefit from RAI, so the direction of care was transferred to a palliative approach. After extensive physiotherapy and occupational therapy input the patient was discharged home with ongoing allied healthcare and community palliative care input.

\section{Discussion}

Thyroid carcinomas as a group are an uncommon cancer and account for approximately $1.5 \%$ of cancers in adults [5]. Of the subtypes previously mentioned, FTC accounts for approximately $10-30 \%$ of cases and is classed as a differentiated cancer $[2,6]$. FTC is characterized by an indolent growth rate and can remain without signs and symptoms beyond metastatic dissemination. Despite this, FTC usually presents as a single thyroid nodule and patients diagnosed at this stage without metastatic spread have an excellent 10 -year survival quoted as $85 \%$ [2]. The incidence of FTC is higher in endemic areas of iodine deficiency or endemic goiter [5]. In the 1990s iodine deficiency was a major problem in Fiji with almost 50\% showing signs of goiter caused by this deficiency [7]. Of note, the patient discussed in this case was of Fijian background and had lived in Fiji the majority of his life.

A key component of the pathophysiology of FTC is its ability for early vascular invasion and hematogenous spread to bone and lung. It is estimated that $10-25 \%$ of newly diagnosed cases of FTC present with metastatic disease [8-11]. The 
primary patient complaint of bone metastases is usually pain. Although bony metastases are a known complication of FTC, presentation due to cord compression is extremely rare. As a slow growing malignancy, this represents late-stage disease. Patients presenting with signs and symptoms of spinal cord compression due to a lesion can pose a diagnostic conundrum, whereby thyroid cancer may not be investigated before a histological result is back. This case demonstrates that along with other cancers that readily metastasis to bone, such as breast, lung and prostate, thyroid cancer must also be considered early, clinically assessed and treated appropriately [12].

Surgical resection is advised as the initial treatment for differentiated thyroid cancers such as FTC [13]. The choice of lobectomy versus total thyroidectomy depends on factors including size of nodule, multifocal disease and risk factors such as radiation exposure and family history [13]. If diagnosed with metastatic disease, the patients are usually discussed in an MDT setting, with additional therapies such as RAI and thyroid hormone suppression being offered to the patient as they have shown to be beneficial to patients with metastatic disease, age $>45$ or large primary tumors $>4 \mathrm{~cm}$ [13]. Ten-year survival for these patients has been quoted as 26-60\% [14]. Therefore, most patients with metastatic disease will initially undergo thyroidectomy and undergo subsequent RAI ablation several weeks later. The result of the orthopedic MDT had ruled out the possibility of surgical resection of the large metastatic iliac mass, which had already undergone external beam radiation and cryoembolization. This meant that RAI would not be effective and so it was not offered to the patient.

A specific issue for the clinicians in this case was the management of the patient's neurological deficits. Regaining some function to be able to defecate and void urine without assistance as well as the upper body strength development to help with movement increases quality of life in patients, even if no further active measures are indicated for their malignancy. This is best achieved through a spinal rehabilitation facility. Despite compromised survival, patients with malignant spinal cord compression make clinically significant functional gains and exhibit favorable discharge outcomes following inpatient rehabilitation [15].

In conclusion, this case highlights the rarity of malignant cord compression from metastatic FTC, its difficulty in management and barriers to discharge. In tandem with other reported cases, this patient presented without significant back pain, symptoms of thyroid disease but with signs of metastatic cord compression. As discussed, these aspects are most likely due to the indolent cancer growth coupled with early vascular invasion and spread. Despite emergent spinal surgery and thyroidectomy, due to the patient's significant metastatic disease, completion treatment with RAI was not possible. This resulted in a cancer that usually has a good survival even when metastases are present, to a terminal disease for this patient.

\section{Acknowledgments}

None to declare.

\section{Financial Disclosure}

None to declare.

\section{Conflict of Interest}

None to declare.

\section{Informed Consent}

Informed consent was gained prior to completing the case report.

\section{Author Contributions}

$\mathrm{RP}$ and $\mathrm{KB}$ both contributed to the conception, writing and editing of the case report. NN provided oversight to the case report.

\section{Data Availability}

The authors declare that data supporting the findings of this study are available within the article.

\section{References}

1. Tang J, Kong D, Cui Q, Wang K, Zhang D, Liao X, Gong $\mathrm{Y}$, et al. The role of radioactive iodine therapy in papillary thyroid cancer: an observational study based on SEER. Onco Targets Ther. 2018;11:3551-3560.

2. McNeeley MF, Sabath A, Linnau KF. Follicular thyroid carcinoma presenting as acute cord compression due to thoracic vertebral metastasis. Radiol Case Rep. 2012;7(3):687.

3. McHenry CR, Phitayakorn R. Follicular adenoma and carcinoma of the thyroid gland. Oncologist. 2011;16(5):585593.

4. Dralle H, Machens A, Basa J, Fatourechi V, Franceschi S, Hay ID, Nikiforov YE, et al. Follicular cell-derived thyroid cancer. Nat Rev Dis Primers. 2015;1:15077.

5. Baiao JM, Guimaraes A, Moreira N, Correia JG, Rosenvinge CU, Goncalves D, Calvo MA. Acute paraparesis as presentation of an occult follicular thyroid carcinoma: A case report. Int J Surg Case Rep. 2017;41:498-501.

6. Kim D, Jwa C, Kim K, Kang J. Spinal cord compression as initial presentation of follicular thyroid carcinoma. J Korean Neurosurg Soc. 2007;41:269-271.

7. Micronutrients. Ministry of Health \& Medical Services. [Cited 2020 Aug 25]. http://www.health.gov.fj/iron-deficiency/.

8. Shaha AR, Shah JP, Loree TR. Differentiated thyroid cancer presenting initially with distant metastasis. Am J Surg. 1997;174(5):474-476. 
9. Lin JD, Huang MJ, Juang JH, Chao TC, Huang BY, Chen KW, Chen JY, et al. Factors related to the survival of papillary and follicular thyroid carcinoma patients with distant metastases. Thyroid. 1999;9(12):1227-1235.

10. Eichhorn W, Tabler H, Lippold R, Lochmann M, Schreckenberger M, Bartenstein P. Prognostic factors determining long-term survival in well-differentiated thyroid cancer: an analysis of four hundred eighty-four patients undergoing therapy and aftercare at the same institution. Thyroid. 2003;13(10):949-958.

11. Girelli ME, Casara D, Rubello D, Piccolo M, Piotto A, Pelizzo MR, Busnardo B. Metastatic thyroid carcinoma of the adrenal gland. J Endocrinol Invest. 1993;16(2):139141.

12. Barron KD, Hirano A, Araki S, Terry RD. Experiences with metastatic neoplasms involving the spinal cord. Neurology. 1959;9(2):91-106.
13. Haugen BR, Alexander EK, Bible KC, Doherty GM, Mandel SJ, Nikiforov YE, Pacini F, et al. 2015 American Thyroid Association Management Guidelines for adult patients with thyroid nodules and differentiated thyroid cancer: The American Thyroid Association Guidelines Task Force on thyroid nodules and differentiated thyroid cancer. Thyroid. 2016;26(1):1-133.

14. Iwasaki H, Yamazaki H, Takasaki H, Suganuma N, Sakai R, Nakayama H, Hatori S, et al. Treatment outcomes of differentiated thyroid cancer with distant metastasis improve by tyrosine kinase inhibitors. Oncol Lett. 2019;17(6):5292-5300.

15. Fortin CD, Voth J, Jaglal SB, Craven BC. Inpatient rehabilitation outcomes in patients with malignant spinal cord compression compared to other non-traumatic spinal cord injury: A population based study. J Spinal Cord Med. 2015;38(6):754-764. 